\title{
Arthur Menzel (1844-1878), einer der liebsten und tüchtigsten Schüler und der treuesten Freunde Billroths, Primarchirurg in Triest
}

Von Loris Premuda

Seit einigen Jahren befasse ich mich mit einer Reihe von Untersuchungen über das Eindringen der Lehren und Errungenschaften der Wiener medizinischen Schule des 19. Jahrhunderts in Italien und über die kulturelle Rolle der Personen, die die Vermittlung zwischen der mitteleuropäischen und italienischen Wissenschaft vollzogen haben ${ }^{1}$. Im Laufe dieser Untersuchungen, die sich notwendigerweise und selbstverständlich - nicht nur aus geographischen, sondern auch aus politischen Gründen - auf die zwei Pole Padua und Triest beziehen, die am stärksten in diesen $\mathrm{V}$ organg verwickelt sind, ergaben sich nicht selten Elemente, die bemerkenswert und geschichtlich interessant sind. Leider waren diese wegen der heiklen politischen Lage der in umstrittenen Grenzgebieten gelegenen, zuerst dem österreichisch-ungarischen Reich angehörenden und seit 1866 beziehungsweise 1918 der italienischen Jurisdiktion überlassenen Städte in Vergessenheit geraten. Die Ereignisse und Personen im Rahmen einer von nationalen Forderungen und politischen Diskussionen bewegten Periode um die Jahrhundertwende waren vom Glück weniger begünstigt: sie sind von beiden Seiten aus entgegengesetzten, obwohl gleichartigen Gründen vergessen worden.

Die Bedeutung und Wichtigkeit der wissenschaftlichen Werke und der Lehrtätigkeit von Theodor Billroth, die die Grenzen Europas bei weitem überschreiten, sind unter anderem von Henry E. Sigerist ${ }^{2}$ und von Erna Lesky $^{3}$ vorzüglich dargestellt worden. Jede weitere Hervorhebung ist überflüssig. Unter der ersten Schülergeneration des berühmten Meisters in Wien stehen in erster Linie Vinzenz Czerny (1842-1916), Karl Gussenbauer (1842-1903), Robert Gersuny (1844-1924) und Arthur Menzel, mit dem wir uns befassen werden.

Arthur Menzel wurde am 21. August 1844 in Görz als Sohn von Wenzel Joseph Menzel, dem Direktor des k.k. Gymnasiums jener Stadt und später dessen von Triest, geboren. Er hatte einen fünf Jahre jüngeren Bruder Oskar, der Angiolina Bussi heiratete, die aus einer nach Triest übersiedelten lombardischen Familie stammte und ihm zwei Kinder schenkte: Ernesto, 


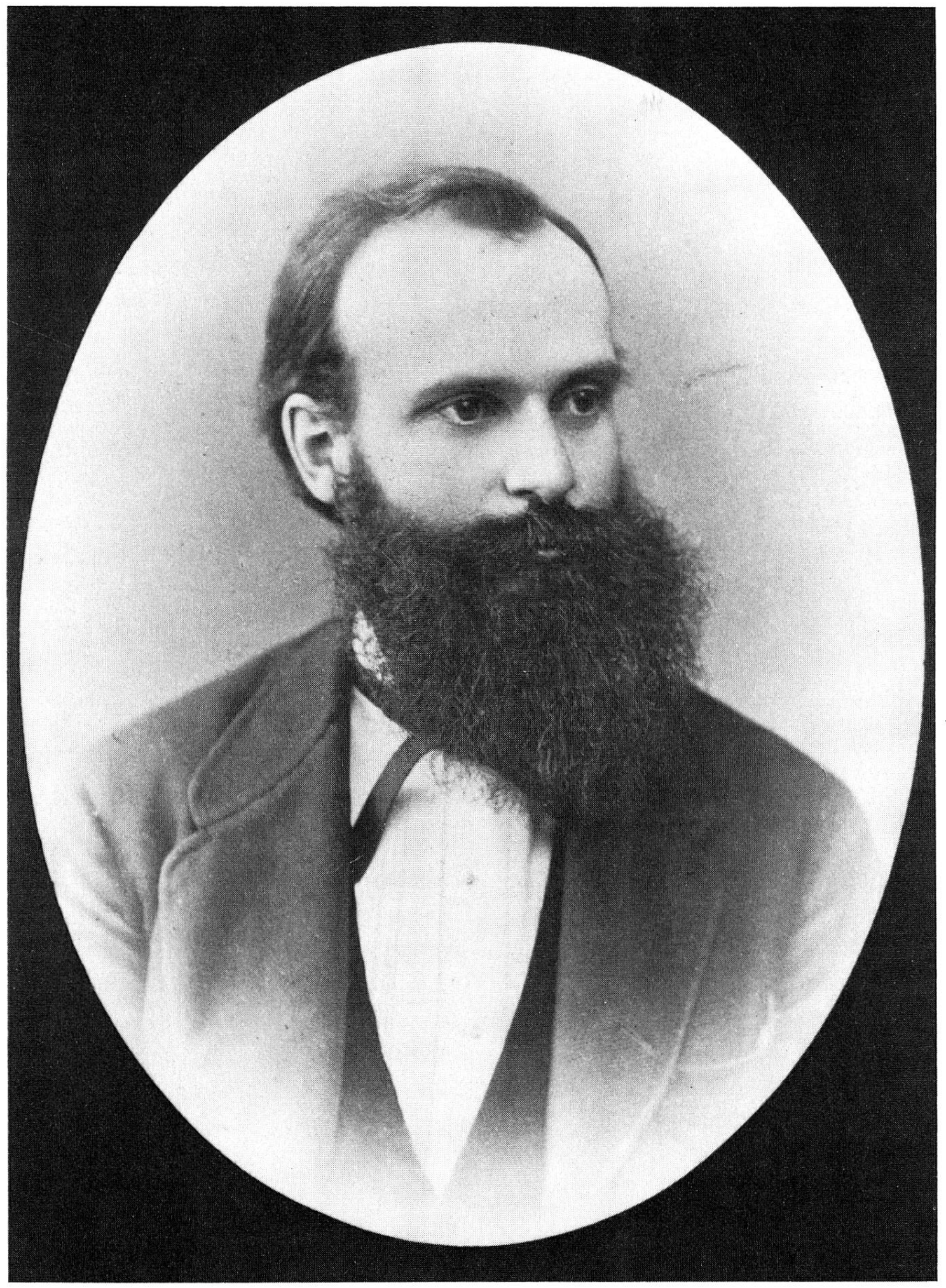

Abb. 1. Arthur Menzel im Alter von 30 Jahren 
der um 1900 Arzt und Operationszögling unter Gussenbauer wurde ${ }^{4}$, und Lucy, die sich mit einem Carmelich vermählte.

Nachdem Arthur Menzel in Triest das k. k. Gymnasium besucht und das Abitur bestanden hatte, erhielt er am Ende des Schuljahres 1861 ein Zeugnis der Vorzugsklasse. Unter den in der achten Klasse studierten Fächern erwähnen wir die Lektüre von zwei Büchern der Annales von Tacitus, einer Anthologie der Carmina, der Epistulae und der Satirae des Horaz, einiger Gesänge der Ilias und der Gorgias von Platon, Elemente der Wahrscheinlichkeitsrechnung, empirische Psychologie und - was nicht unwichtig ist Hodegetik des akademischen Studiums ${ }^{5}$. Einer seiner Mitschüler war Lorenz Lorenzutti (1843-1912), der später auch in Wien mit ihm studiert hat und dann Primarius der VI.Abteilung für innere Medizin an demselben städtischen Krankenhaus von Triest wurde, an dem Menzel seine kurze Karriere beenden sollte.

Im akademischen Jahr 1861-62 begann Menzel sein Medizinstudium an der Universität Wien, wo er dann 1867 promovierte. Unter seinen Lehrern hatte er die berühmtesten Namen der Medizin jener Epoche: Joseph Hyrtl (1811-1894) in Anatomie, Ernst Brücke (1819-1892) in Physiologie, Carl von Rokitansky (1804-1878) in Pathologischer Anatomie und Johann von Oppolzer (1808-1871) in Medizinischer Klinik.

Menzels Promotionsjahr stimmt mit dem Berufungsjahr Theodor Billroths von Zürich nach Wien überein. Er näherte sich sofort dem berühmten Meister, indem er sich am Chirurgischen Institut für den Lehrkurs 1867-1869 ${ }^{6}$ einschrieb, und zwar noch bevor in der Organisation. die von Billroth $^{7}$ beantragten weitgehenden Veränderungen verwirklicht wurden. Billroths Schüler Eduard Albert (1841-1900) und Vinzenz Czerny waren zusammen mit Menzel. In der ersten Etappe seiner chirurgischen Laufbahn war er Operationszögling. Ende 1869 wurde er bereits klinischer Assistent an der zweiten chirurgischen Klinik der medizinischen Fakultät Wien. 1872 habilitierte er sich als Dozent für Chirurgie.

Am 5. März desselben Jahres schrieb Billroth an Dr. Brettauer, den Präsidenten des städtischen Krankenhauses zu Triest, um Arthur Menzel als Kandidaten für das Amt des chirurgischen Chefarztes jener Stadt vorzuschlagen, worum sich offensichtlich, wie aus dem Brief hervorgeht, auch einige Schüler von Johann Dumreicher (1815-1880), dem Leiter der ersten chirurgischen Klinik in Wien und Triestiner von Geburt, bewarben. Billroth beendete seinen Brief mit den Worten: «Über so ungeheuerliche Anpreisungen, wie die von Dumreicher erwähnten, kann ich freilich nicht disponieren; 


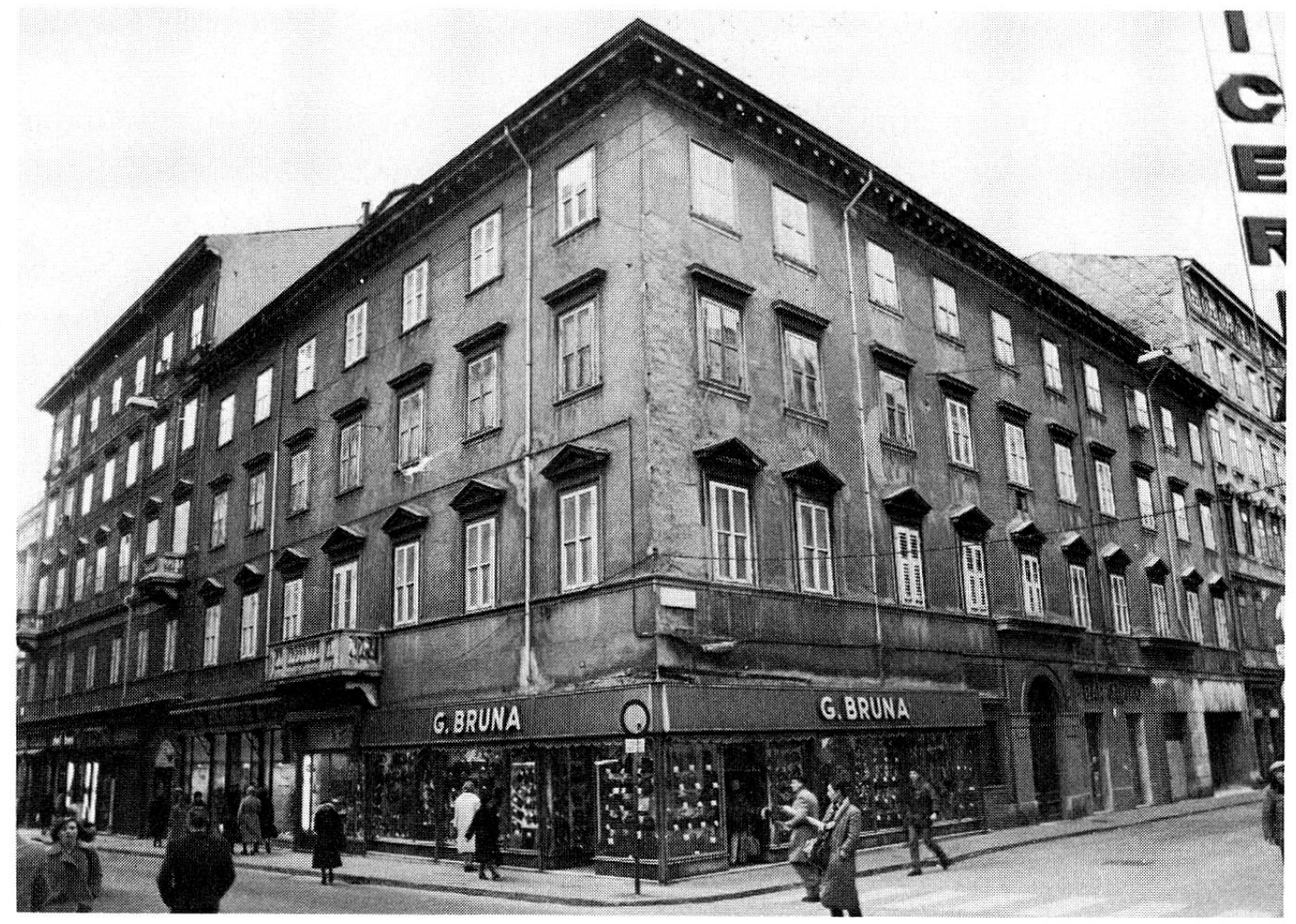

Abb.2. Das Haus im Zentrum von Triest, wo Menzel als Arzt wohnte

ich hoffe jedoch einen anderen Ton zu treffen, der möglicherweise wirksamer ist. Hoffen wir für unseren Schützling das Beste.» ${ }^{8}$ Am 1. August 1872, erst 28 Jahre alt, übernahm Menzel die IV.chirurgische Abteilung des Triester Krankenhauses. Er wurde damit Nachfolger von Giovanni Cappelletti (1808-1872), der unter Dupuytren (1777-1835) in Paris, an der Londoner Schule und unter Carl Ferdinand von Graefe (1787-1840) in Berlin studiert hatte.

Die praktische, wissenschaftliche und humanitäre Tätigkeit des jungen Primarius während seines Aufenthaltes in Triest ist bemerkenswert. Seine Mitarbeiter waren Ventura Romanin (1844-1924), ein Triestiner und ehemaliger Assistent von Dumreicher (März 1869 bis Oktober 1871), später in Triest ein Pionier der Röntgendiagnostik und der Röntgentherapie ${ }^{9}$, Antonio Sbisà (1846-1905), ebenfalls aus der Wiener Schule, Ugo de Porenta (1849-1902), Ottaviano Baldini (1852-1883) und der Zürcher Theodor Escher (1847-1923), Schüler von Edmund Rose (1836-1917) in Zürich sowie kurze Zeit von Billroth in Wien und Nachfolger Menzels nach dessen Tode. 
Lodovico Brunetti (1813-1899), Schüler von Joseph Wattmann (1789-1866) und Assistent von Rokitansky, der 1855 auf den gerade errichteten Lehrstuhl für pathologische Anatomie an der Universität Padua berufen wurde, aber seit jeher in die Chirurgie verliebt war und nebenher in der chirurgischen Abteilung von Menzel in Triest verkehrte, behauptete mit Recht, daß Menzel «nicht nur von seinen Hilfsärzten, ... sondern auch von den Hilfsärzten der anderen Abteilung assistiert wurde, denn alle eilen herbei, wo es etwas zu lernen gibt». ${ }^{10}$

Menzel wohnte im Zentrum von Triest, und zwar in der ersten Etage eines zu jener Zeit ziemlich neuen Wohnhauses. In seinem Privatambulatorium, das von den Einwohnern Triests und der umliegenden Länder sehr besucht war, praktizierte er von $15-16$ Uhr nachmittags ${ }^{11}$.

Der Winter 1877-78 stellte, wie es scheint, die Konstitution Menzels hart auf die Probe. Trotzdem begab er sich im Mai nach Berlin. Er wollte dort am Chirurgen-Kongreß teilnehmen, «um neue Lehren und neue Anregung zum Wissen, sowie gleichzeitig Ruhe und Zerstreuung zu suchen». ${ }^{12}$ Kurz nach

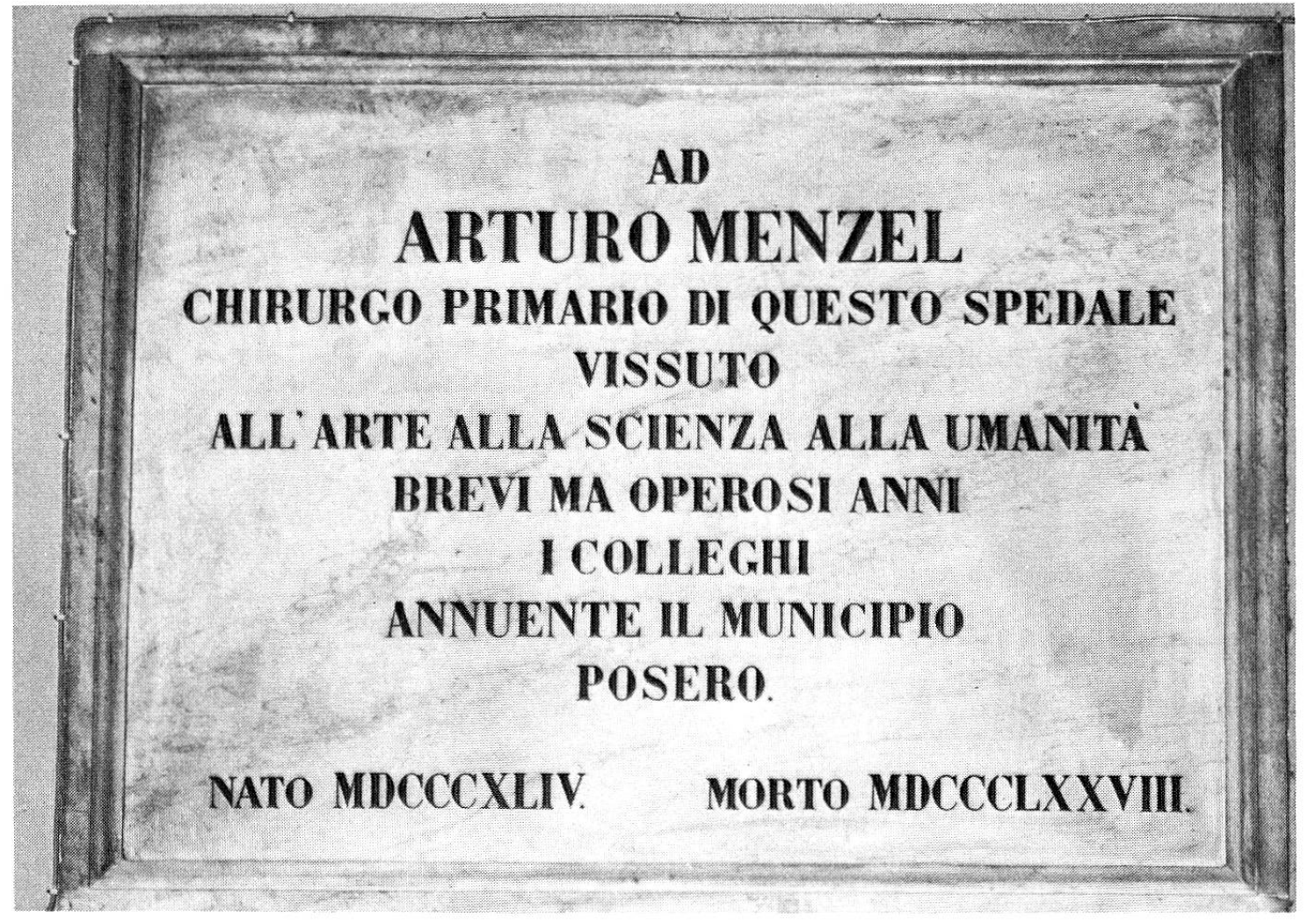

Abb.3. Die Gedenktafel rechts im Vorraum des städtischen Krankenhauses (heute: Ospedale Maggiore) in Triest 
Wien wurde er von einem leichten Bluthusten befallen. Er reiste weiter; aber als er in Berlin ankam, konnte er sich nicht zum Kongreß begeben, sondern mußte ins Augusta-Hospital eingeliefert werden. Dem Bruder, der an sein Bett eilte, sagte er wörtlich: «Wenige Tage, bevor ich Triest verließ, träumte ich davon, in Berlin zu sterben. Wenn ich an Träume geglaubt hätte, wäre ich zu Hause geblieben.» ${ }^{13}$ Die fürsorgliche Pflege der Kollegen und Freunde in Berlin konnte ihm nicht helfen. Am 12. Mai 1878 starb Menzel an der Krankheit, die ihn befallen hatte. Am 14. Mai verbreitete sich in Triest die Nachricht von dem frühen Tode des Chirurgen. Eine Zeitung schrieb: «Die Wissenschaft verliert einen ihrer gewissenhaftesten und intelligentesten Vertreter und die Menschheit einen sehr tüchtigen Chirurgen.» ${ }^{14}$ Die «Associazione Medica Triestina», deren zweiter Vizepräsident er gewesen war, ehrte den Verstorbenen bei der Beerdigung und in Gedenkfeiern; sie hob die Notwendigkeit hervor, daß «die IV. Abteilung des Krankenhauses und unsere Einwohnerschaft im Nachfolger von Dr. Arthur Menzel jene Autorität finden müssen, die mit dem Namen des letzteren vereint werden kann».. ${ }^{15}$

Billroth nahm am Schmerz des Triestiner Ärztevereins mit einem bewegten Brief an Dr. Brettauer teil, und zwar am Tage nach dem Tode seines Schülers, als er die telegraphische Nachricht aus Berlin erhielt. Nachdem er die im Titel dieser Arbeit angeführten Worte gebraucht hatte, um Menzel zu beschreiben, hob er außerdem hervor: «Es war ein Mensch mit einer so schönen anima candida, wie man nicht Viele findet, strebsam, human, ganz erfüllt von Pflichtgefühl.» ${ }^{16} \mathrm{Zwei}$ Tage später, am 15. Mai, ohne die Möglichkeit aus den Augen zu verlieren, daß unter den Schülern des Verstorbenen ein Nachfolger gefunden werden könnte, schlug er Brettauer Robert Gersuny als Kandidaten vor, indem er hinzufügte: «Menzel selbst würde mir darin beistimmen.» ${ }^{17}$ Diesmal drehte sich das Rad des Schicksals anders: Menzels Nachfolger wurde Theodor Escher, der Assistenzarzt des Verstorbenen.

In einem begeisterten Nekrolog unterstrich Brettauer unter anderem die Wichtigkeit von Menzels Vermittlung zwischen der Wiener medizinischen Kultur und der italienischen Medizin. Menzel hatte die deutsche und italienische Sprache vollkommen beherrscht. Weiter erzählte Brettauer: «Ich erinnere mich, daß vor zwei Jahren, als ich mit Menzel eine Reise in Italien unternahm und gerade damals Porta von Pavia verstorben war, in allen Städten, von Venedig bis Neapel, mir die Frage gestellt wurde, ob Menzel sich um jenen Lehrstuhl beworben hätte.» ${ }^{18}$

Am Ende dieses kurzen Lebensbildes entsinne ich mich des Inhaltes und des Wesens eines wertvollen Buches von Paul Diepgen: «Unvollendete». ${ }^{19}$ 
Der deutsche Medizinhistoriker hat darin die Biographien junger Ärzte vereinigt, die jung, in der vollen Entfaltung ihrer wissenschaftlichen Tätigkeit gestorben sind. In ihre Reihe könnte man auch Menzel aufnehmen.

Menzel trat zum erstenmal wissenschaftlich auf mit der Mitteilung eines klinischen Vortrages, der am 5. Juni 1868 gehalten wurde. Hierauf beschrieb er in der Wiener medizinischen Wochenschrift den Inhalt einer klinischen Vorlesung Billroths über «Chloroformwirkung und Chloroformtod».. ${ }^{20}$ Die Anästhesie war eine der brennendsten und faszinierendsten Fragen der Chirurgie jener Zeit. Die Chloroforminhalationsnarkose bei Geburten und Operationen war durch den Engländer James Young Simpson (1811-1870) 1847 eingeführt worden. Frankel berichtet: "In a surprisingly short time chloroform was the predominant anesthetic in Germany", und "Binz has reported from his own experiences during the Austro-Prussian war in 1866 and the Franco-German war (1870-71) that only chloroform was used for general anesthesia." 21 Wie Menzel darlegt, erklärte Billroth: «Gefahr kann die Chloroformnarkose in allen Stadien bringen.» ${ }^{22}$ Aber: «Daß die Anwendung des Chloroformkorbes das beste, relativ sicherste und billigste Verfahren der Inhalation ist, darüber herrschen keine Zweifel mehr ...; man braucht jetzt wohl überall Chloroform allein.» ${ }^{23}$ Ein Fall von Chloroformtod darf den Chirurgen nicht entmutigen: «Lassen Sie sich durch solche Unglücksfälle, vor denen Sie ein gütiges Geschick in Ihrer künftigen praktischen Thätigkeit bewahren möge, nicht die Freude an unserer herrlichen Wissenschaft und Kunst verkümmern!» ${ }^{24}$ Auf diesen Ton war man in Wien an der zweiten chirurgischen Klinik am Ende der sechziger Jahre gestimmt. Bereits Mitte 1870 wird uns Menzel selbst in den Berichten über seine chirurgischen Eingriffe immer häufiger davon berichten, daß er nun in der Anästhesie zur Einführung der englischen Mischung übergegangen sei (3 Teile Chloroform, 2 Teile Äther und 1 Teil Alkohol). Auf klinisch-operativer Ebene sind dies Jahre großer Spannungen; die Ergebnisse von immer neuen und verschiedenen Erfahrungen folgen sich mit bedrängender Geschwindigkeit.

Im April 1869 griff Menzel, damals noch Operationszögling, mit einer Experimentalarbeit, die er zusammen mit seinem Landsmann Dr. Hermann Perco, k.k. Sekundararzt im allgemeinen Krankenhaus, durchgeführt hatte, in eine Debatte ein, die einerseits biochemisch und pharmako-therapeutisch interessant ist, andererseits eine praktische Beziehung zur prä- oder postoperativen Behandlung von Patienten hat, die sich wegen schwerer 
Erkrankungen des Verdauungstrakts einer Operation unterziehen müssen. Die Frage, die die zwei Forscher beantworten wollten, lautete: «Wenn man den Entschluß faßt, Nahrungsmittel subkutan zu injizieren, werden diese dann resorbiert?» Als Versuchstiere wurden Hunde genommen und als einzuspritzende Mittel Fette, Milch, Zucker und Eiweiß (von den Fetten Mandelöl, Olivenöl, Lebertran). Es ergaben sich keine Beschwerden, nicht einmal im einzigen Fall eines Mannes, der infolge einer Caries vertebrarum schwer verfallen war und auf Anordnung Billroths der Behandlung unterzogen wurde. Die Resorption fand innerhalb 36-48 Stunden statt ${ }^{25}$. Die Autoren erwähnen, daß sie durch mündliche Mitteilung von den von Stricker und Oser durchgeführten subkutanen Injektionen von Pepton erfahren hatten. Aber wie man mit Recht annehmen kann, sind ihre systematischen Erfahrungen die ersten auf diesem Gebiet. Die Verordnung der Subkutanspritze zur Therapie reicht allerdings in das Jahr 1853 zurück und ist mit dem Namen von Charles Pravaz (1791-1853) verbunden. Die Einführung von Wasser oder Salzlösungen in das Unterhautzellgewebe im Falle von Cholera versuchte man ungefähr um dieselbe Zeit oder etwas später. Wir finden hier Namen wie Buchanan (1798-1882) ${ }^{26}$ und Cantani (1837-1893) ${ }^{27}$ unter den ersten Befürwortern der sogenannten subkutanen Infusion. Menzel und Perco beenden den Bericht über ihre Erfahrungen mit Bescheidenheit und objektiver Hoffnung: «Durch die mitgetheilten Versuche ist es erwiesen, daß die erwähnten Substanzen vom Unterzellgewebe bei Hunden ohne entzündliche Erscheinungen resorbirt werden. Es wird Gegenstand weiterer Versuche sein müssen, ob beim Menschen die gleichen Resultate zu erzielen sind, sowie ob Hunde durch subkutane Injektionen von Nahrungsmitteln allein eine Zeit lang dem Leben erhalten werden können.» ${ }^{28}$

Menzel erweiterte sein Wissen im Bereich der experimentellen Pathologie, indem er eine neue experimentelle Studie über die Urin-Infiltration unternahm. Den Ausgangspunkt bildeten Erfahrungen von Gustav Simon (1822-1876), der als Autor der neuen Operation der Blasenscheidenfistel (1852-54) besser bekannt ist. Menzel nahm sich vor, die Einwirkung des Urins auf das Zellgewebe festzustellen ${ }^{29}$. Billroths Einfluß auf seine Schüler ist dabei offenkundig: es ging darum, der Klinik durch Vorproben im Bereiche des Labors experimentell und propädeutisch zu dienen. Menzel wollte die Gültigkeit des zu seiner Zeit noch herrschenden Grundsatzes überprüfen, daß der infiltrierte Urin ausnahmslos den Brand der Gewebe bedinge ${ }^{30}$. Er unternahm Versuche mit saurem Harn und mit ammoniakalischem Harn und kam nach anatomisch-pathologischen, chemischen und 
klinischen Untersuchungen sowie nach der Überprüfung der Fachliteratur und nach der Beratung mit Persönlichkeiten wie Leopold von Dittel (1815-1898), dem Begründer der Wiener Urologie, zu der Schlußfolgerung: «Saurer Urin bedingt an und für sich keine Gangrän» ${ }^{31}$, aber umgekehrt: «Es liegt somit der Beweis vor, daß auch das kohlensaure Ammoniak in jeder Menge, wie es sich höchstens im normalen Harne bilden kann, im Stande ist, Jauche und Gangrän zu erzeugen.» ${ }^{32}$

Aus demselben Jahr 1869 stammen Versuche «Über die septischen Eigenschaften des frischen Wundserums» ${ }^{33}$ Sie wurden an Hunden durchgeführt. Dabei wurden die Mechanismen der Eiterbildung untersucht. Man befand sich in der Übergangszeit von der humoralen zur streng naturalistischen Interpretation der pathologischen Erscheinungen. Nur zwölf Jahre vorher hatte Pasteur (1822-1895) die endgültige Widerlegung der spontanen Entstehung der Bakterien geliefert, und sechzehn Jahre vorher hatte er über die parasitäre Erkrankung der Seidenraupe geforscht. Zu dieser Gruppe von Untersuchungen gehören 1870 auch Menzels «Experimentelle Studien über die Tuberkulose» ${ }^{34}$, die 12 Jahre vor der Entdeckung der Tuberkelbazillen durch Robert Koch (1843-1910) mit der einprägsamen und prophetischen Behauptung endeten: «In diesem Sinne ist es, glauben wir, keine bloße Hypothese, wenn man sagt: es gibt einen spezifischen Tuberkelstoff, die käsige Masse enthält das Seminium der Tuberkulose.» ${ }^{35}$

In der ersten Hälfte des 19. Jahrhunderts erfolgte eine starke Wiederaufnahme der Forschung in der Knochenpathologie und eine darauffolgende Entwicklung in der Chirurgie der Bewegungsorgane. Dupuytren (17771835) hatte die Frage nach der Kallusbildung neu gestellt ${ }^{36}$. Menzel fügte sich dieser Forschungsrichtung ein. In einem Brief vom 2. März 1871 an Prof. Gurlt in Berlin hob Billroth zwei Arbeiten seines Schülers besonders hervor, die von ihm ausgewählt worden waren, um sie zur Veröffentlichung dem Verleger Hirschwald zu schicken: die erste über den Effekt der Gelenkruhe und die zweite über die Kieferresektion ${ }^{37}$.

In Nachahmung der von Léopold Ollier (1830-1900) ${ }^{38}$ und von Julius Wolff (1836-1902) ${ }^{39}$ durchgeführten Versuche nahmen zuerst Billroth allein in Zürich und etwas später Janny und Menzel zusammen mit ihrem Lehrer in Wien das wichtige Thema der «Osteoplastischen Versuche» ${ }^{40}$ an Hunden wieder auf. Ausgeführt wurden die Ablösung von Periostlappen, die Entfernung von Knochenteilen samt dem Periost, die Entfernung von Knochenteilen unter Zurücklassung des Periosts und zuletzt die Replantation und Transplantation vollständig ausgelöster Knochenpaarstücke. Die Schluß- 
folgerung stand deutlich und klar im Bereich des strengsten experimentellen Denkens: «Man muß diese Operationen noch häufiger machen und die Schlußresultate derselben weiter kennen lernen, bevor man sich darüber ein Urtheil bilden kann.» ${ }^{41}$

In diesem Bereich hat Menzel noch weitergearbeitet. Eine Abhandlung erläutert die Erkrankung der Gelenke bei dauernder Ruhe derselben ${ }^{42}$, eine andere die Behandlung der Unterschenkelfrakturen ${ }^{43}$. Die Gelenksruhe reicht nach seiner Meinung nicht aus, die Erkrankung der Gelenke hervorzurufen, sondern es ist der gegenseitige Druck der Gelenkfacies notwendig. Das Material des Triester Krankenhauses war reich an Traumen. Es ermöglichte Studien über die Behandlungsverfahren hauptsächlich der Fraktur mit Dislokation. Die restlichen Arbeiten stützen sich auf einzelne Fälle (im Einklang mit zahlreichen klinischen Beiträgen jener Epoche): eine Kniegelenkresektion ${ }^{44}$, ein Rippenchondrom ${ }^{45}$, ein Osteofibrom des Unterkiefers ${ }^{46}$, ein fibröser Polyp der Schädelbasis (Exstirpation mit Resektion des Oberkiefers) ${ }^{47}$, eine isolierte Infraktion der Tibia bei einem Erwachsenen ${ }^{48}$, eine Uranoplastik ${ }^{49}$, eine angeborene Sakralgeschwulst ${ }^{50}$. Eine von Billroth und Menzel durchgeführte statistische Untersuchung über die Häufigkeit der Caries in den verschiedenen Knochen und über die Kombination chronischer Knocheneiterungen mit Tuberkulose ergänzt das Bild der wissenschaftlichen Produktion im Bereich der Osteopathien ${ }^{51}$.

Die erste Ovariotomie war wahrscheinlich jene, die von Ephraim McDowell (1771-1830) durchgeführt wurde. Die erfolgreiche Reihe der Ovariotomien begann 1858 mit den Operationen von Thomas Spencer Wells (1818-1897). So hatte Menzel bereits 1869 in der Klinik Billroths die Ergebnisse von drei Ovariotomien beschrieben. Auf dieses so aktuelle Thema sollte er später noch zurückkommen, als freier Primarchirurg, und zwar mit einer Mitteilung über einen «Fall von spontaner Berstung eines Cystovariums». ${ }^{52}$ Die Krankheit war als «Cirrhosis hepatis» diagnostiziert und zu lange in der Medizinischen Abteilung behandelt worden; erst einen Tag vor dem Tode wurde die Frau in die Abteilung Menzels gebracht. Die sichere Diagnose ergab sich erst aus dem Sektionsbefund, denn «in unserem Fall waren aber die Symptome der Erkrankung ganz allmälig aufgetreten»..$^{53}$ Mit Recht unterstreicht der Autor die Gültigkeit des Gedankens von Wells: «Die Berstung einer Cyste ist nicht ein Hinderniss für die Ovariotomie, im Gegentheil sie ist ein Grund, die Operation ohne Aufschub vorzunehmen.» ${ }^{54}$ Erfreulich waren dagegen die von Menzel erzielten Ergebnisse in zwei glänzend beschriebenen Fällen: Polycyste des linken Ovariums; totaler 
Verfall der Gebärmutter; vollkommene Heilung durch Ovariotomie. Polycyste des rechten Ovariums; Ovariotomie; großer Abdominalabszeß; Drainage; Symptome von Pyohämie; Heilung ${ }^{55}$.

Das Jahrzehnt von 1865 bis 1875 erlebte eine hohe Blüte der Chirurgie. Ihre Kennzeichen waren die Verbesserung der Narkosetechnik, die Begründung der Antisepsis durch Joseph Lister (1827-1912) und die Einführung der künstlichen Blutleere durch Friedrich Esmarch (1823-1908). Unter allen drei Gesichtspunkten nimmt der Triestiner Primarchirurg eine avantgardistische Stellung ein. Freundlich, aber bestimmt klingt 1877 seine an die verschiedenen Krankenhäuser und Chirurgen gerichtete Aufforderung, «über die Narkosen eine genaue Statistik zu führen». Er wiederholt, daß er nun eine Mischung von 3 Teilen Chloral, 2 Teilen Äther und einem Teil Alkohol brauche und daß man aus dem Vergleich zwischen den verschiedenen Statistiken in wenigen Jahren große Vorteile ziehen könne ${ }^{56}$. Am 4. Juni 1873 wandte er zum erstenmal in Triest die Methode von Esmarch an, und zwar bei einem Eingriff an einem Mann von 65 Jahren, dem ein Sarkom an der inneren Seite der oberen Hälfte des linken Oberschenkels entfernt werden mußte ${ }^{57}$. «Die Operation dauerte mit Einschluß der Narkose dreiviertel Stunden, und der Kranke verlor keinen Tropfen Blut. Die Operation wurde auf dieselbe Weise durchgeführt wie an der Leiche.» ${ }^{58}$

Die ersten Ergebnisse über die antiseptische Behandlung der Wunden im nordöstlichen Italien waren jene von Angelo Minich (1817-1893), der bereits am 2.Januar $1876^{59}$ seine reichhaltige Monographie in Druck gab. Er berichtete darin über persönliche Erfahrungen im Gebrauch der Karbolsäure, wie sie von Lister angeraten wurde, und des Sodasulfits, das er selber vorgeschlagen hatte.

Anfangs 1876 führte Menzel systematisch Listers Methode ein ${ }^{60}$. In den drei vorangegangenen Jahren war die auf Sepsis-Pyohämie zurückzuführende Sterblichkeitsrate um $10 \%$ gesunken. Es müsse jedoch hervorgehoben werden, daß $50 \%$ der in der Abteilung aufgenommenen Kranken von Verletzungen verschiedener Art befallen gewesen seien. Im Jahre 1876 verringerten sich die Todesfälle sofort um 7,73\% ${ }^{61}$. Das einzige Hindernis für die Verbreitung des Verfahrens waren die hohen Kosten der Behandlung. Aber nach Menzels Meinung sollte es keine Bedenken für die Anwendung auf breiter Ebene geben ${ }^{62}$. Die ausgezeichneten Ergebnisse des Triestiner Primars werden vom bereits erwähnten Brunetti, der Gast von Menzel war, bestätigt. Brunetti wurde der Vorkämpfer für die Verbreitung der Methoden von Lister im übrigen Italien ${ }^{63}$. Etwas später hat Ruggi (1844-1925), 
Chirurg aus Bologna, Menzels Verdienste auf diesem Gebiet hervorgehoben ${ }^{64}$.

Dem Verfasser dieses Artikels genügt es, der medizinhistorischen Welt einen der hervorragendsten, aber weniger bekannten Schüler Billroths vorgestellt zu haben und auf dokumentierte Weise Menzels Stellung als Vermittler der Chirurgie Billroths in Italien gezeigt zu haben. Für das Urteil über sein Werk überlassen wir seinem angesehenen Lehrer das Wort: «Auf Czerny, Menzel, Gussenbauer, Winiwarter, Mikulicz, Wölfler, Klotz, Frisch darf ich wohl stolz sein. Sie setzen meine Ideen und meine Arbeit fort.» ${ }^{65}$ Und anderswo: «Das soll mir Einer nachmachen, solche Söhne wie Sie [Dr. Gersuny], Frisch, Barbieri, Czerny, Gussenbauer, Winiwarter, Wölfler, Mikulicz, Menzel, Steiner, Hacker, Salzer mit dem Kebsweib Scientia chirurgica zu zeugen, Jeder in seiner Art ein Capital-Kerl!! «Nur die Lumpe sind bescheiden!> $)^{66}$

\section{Anmerkungen}

1 Über dieses Thema habe ich mehr als ein Dutzend Arbeiten veröffentlicht: hier beschränken wir die Zitierung auf drei Sammelberichte: Die vermittelnde Funktion von Triest für die Verbreitung des medizinischen Denkens der Wiener Schule in Italien, in: Wien und die Weltmedizin, hrsg. von Erna Lesky, Wien/Köln/Graz 1974, S.99-115; The influence of the Nineteenth Century Vienna School in Italian Medicine: the roles of Padua and Trieste (im Druck: Baltimore); L'asse Vienna - Padova nella medicina dell'ottocento e $i$ suoi riflessi sul piano didattico e scientifico (im Druck bei der Accademia Patavina di S.S. L. L. A. A.).

${ }^{2}$ H. Sigerist, Große Ärzte, München 1932; darin: Theodor Billroth, S. 280-284.

${ }^{3}$ E. Lesky, Billroth als Mensch und Arzt. Dtsch. Mediz. Journal 17 (1966), H.24, S. 739-743.

${ }^{4}$ Die Zentenarfeier des Operateur-Institutes der Wiener Medizinischen Fakultät, Wien und Leipzig 1907, S. 86.

5 Programm des k.k. Gymnasiums in Triest veröffentlicht am Schlusse des Schuljahres 1861, Triest 1861, S.89f. und 108.

${ }^{6}$ Op. cit. in Anm.4, S. 78.

${ }^{7}$ Ibid., S. 13 ff.

${ }^{8}$ Briefe von Theodor Billroth (hrsg. Dr. Georg Fischer), 9. Aufl., Hannover 1922, S. 122.

${ }^{9}$ E. Horniker, Sulla cura del Tracoma coi raggi Roentgen, Boll. Assoc. Med. Triestina VII (1905), Trieste 1906, S.141-147; L.Premuda, La nascita della radiologia italiana. Cento anni di chirurgia a Trieste 1840-1940. Trieste 1975 (ohne Bezifferung der Seiten).

${ }^{10}$ L. Brunetti, Una riabilitazione chirurgica - Reminiscenze dell'autunno 1876, Padova 1876, S. 6.

${ }^{11}$ Elenco dei medici e chirurghi che esercitano la libera pratica nella città di Trieste e sul territorio, dei proprietari di farmacie nonchè di veterinari ed ippiatri, Trieste 1873, S. 9. 
${ }^{12}$ G. Brettauer, Arthur Menzel, Resoconti Sanitari Osp. Civico Trieste V (1877), Trieste 1878, S.IV-X (hier: V). Auch in der Wien. med. Wschr. 27 (1878) $577 \mathrm{f}$.

13 Ibid.

14 "Il Cittadino» XIII (1878), Trieste, 14 maggio 1878, S.2.

15 Ibid., 21 maggio 1878, S. 2.

16 Op. cit. Anm. 8, S. 182.

17 Ibid., S. 183.

${ }_{18}$ Op. cit. Anm. 12, S. VI.

19 P.Diepgen, Unvollendete, Stuttgart 1960.

${ }^{20}$ Chloroformwirkung und Chloroformtod. Ein klinischer Vortrag, gehalten am 5. Juni 1868 von Professor Dr. Theodor Billroth. Mitgetheilt von Dr. Menzel, Operationszögling, Wien. med. Wschr. 18 (1868) 762-764, 778-780,795-797.

${ }^{21}$ W. K. Frankel, The introduction of General Anesthesia in Germanie, Journal of the History of Medicine and allied Sciences 1 (1946) 612-617 (hier: S. 616).

${ }^{22}$ Op. cit. Anm. 20, S. 778.

${ }^{23}$ Ibid., S. 795.

${ }^{24}$ Ibid., S. 797.

${ }^{25}$ A. Menzel, H.Perco, Über die Resorption von Nahrungsmitteln vom Unterhautzellgewebe aus. Wien. med. Wschr. 19 (1869) $517 \mathrm{f}$.

${ }^{26}$ A. Buchanan, On the injection of the cellular tissue with water, as trial in the year 1848 in the treatment of cholera. Glasgow Med. Journal 2 (1854-55) 358.

${ }^{27}$ A. Cantani, Manuale di Materia Medica e Terapeutica, Vol.1, Milano 1869, S.115; La cura del cholera colle grandi injezioni di acqua alcalina salata. Sonderdruck aus Giornale Int. Sci. Med.5 (1884) 13.

${ }^{28}$ Op. cit. Anm. 25.

${ }^{29}$ A. Menzel, Über die Einwirkung des Urins auf das Zellgewebe. Wien. med. Wschr. 19 (1869) 1349-1353, 1365-1368, 1381-1384, 1397-1399, 1416-1418.

${ }^{30}$ Ibid., S. 1349.

${ }^{31}$ Ibid., S. 1367.

32 Ibid., S. 1417.

${ }^{33}$ A.Menzel, Über die septischen Eigenschaften des frischen Wundserums. Wien. med. Wschr. 19 (1869) $1169 \mathrm{f}$.

${ }^{34}$ Wien. med. Wschr. 20 (1870) 41-44, 57-60, 73-76, 89-91.

${ }^{35}$ Ibid., S.91.

${ }^{36}$ G. Dupuytren, Vorträge über chirurgische Klinik, Bd.2, Berlin 1842.

${ }^{37}$ Op. cit. Anm. 8, S.111f.

${ }^{38}$ L. Ollier, Traité expérimental et clinique de la régénération des os et de la production artificielle du tissu osseux, Paris 1867.

${ }^{39} \mathrm{~J}$. Wolff, Die Osteoplastik in ihren Beziehungen zur Chirurgie und Physiologie. Langenbeck's Arch.f. klin. Chirurgie 4 (1863) 183-294.

${ }^{40}$ Th. Billroth, Janny, Menzel, Osteoplastische Versuche. Wien. med. Wschr.18 (1868) 1533-1535, 1549-1551.

${ }^{41}$ Ibid., S. 1551.

${ }^{42}$ A. Menzel, Über die Erkrankung der Gelenke bei dauernder Ruhe derselben. Arch. f. klin. Chir.12 (1871) 990-998, und: Sulle alterazioni delle articolazioni in seguito a prolungato riposo delle medesime. Il Morgagni 13 (1871) 605-615. 
${ }^{43}$ Idem, Zur Behandlung der Unterschenkelfrakturen. Centralbl.f. Chir.4 (1877), 17-20.

${ }^{44}$ Idem, Ein Fall von Kniegelenkresection, Wschr. d. Ges. d. Wien. Ärzte, Nr. 52.

${ }^{45}$ Idem, Ein Fall von Rippenchondrom. Wien. med. Wschr. 19 (1871) 337-340.

${ }^{46}$ Idem, Ein Fall von Osteofibrom des Unterkiefers. Arch, f. klin. Chir. 13 (1871), 912-914.

${ }^{47}$ Idem, Fibroser Polyp der Schädelbasis. Extirpation mit Resection des Oberkiefers. Arch.f. klin. Chir.13 (1872) 680-682.

${ }^{48}$ Idem, Ein Fall von isolirter Infraktion der Tibia bei einem Erwachsenen. Wien. med. Wschr.24 (1874) 745-747.

${ }^{49}$ Idem, Uranoplastica eseguita in profonda narcosi a capo pendente. Gazzetta Medica Italiana-Lombardia 35 (1876) 8.

${ }^{50}$ Idem, Ein Fall von angeborener Sacralgeschwulst. Arch. f. klin. Chirurgie 22 (1878) 691-697.

51 Th. Billroth und A. Menzel, Über die Häufigkeit der Caries in den verschiedenen Knochen, sowie über die Combination von chronischen Knocheneiterungen mit Tuberculose und anderen chronischen Erkrankungen innerer Organe. Eine statistische Untersuchung. Arch. f. klin. Chirurgie 12 (1870) 341-370.

${ }^{52}$ Wien. med. Wschr.22 (1873) 845-848.

${ }^{53}$ Ibid., S. 847.

54 Ibid., S. 845.

${ }^{55}$ A. Menzel, Zwei Ovariotomien. Wien. med. Wschr.24 (1874) 1017-1020. Das positive Ergebnis der zwei Fälle, die von Menzel durch eine glänzende Operationstechnik behandelt wurden, wird von Robert Michael Olshausen (1835-1915) (Die Krankheiten der Ovarien, Stuttgart 1877, S. 135 und 362) hervorgehoben.

${ }^{56}$ Idem, Zur Statistik der Narkose. Centralbl.f. Chir. 4 (1877) 65 f. (hier: S. 66).

57 A. Menzel, Sul metodo (Esmarch) di eseguire le operazioni alle estremità senza perdita di sangue. Gazzetta Medica Italiana-Lombardia 32 (1873) 185.

${ }^{58}$ Ibid., zweite Spalte.

${ }^{59}$ A. Minich, Cura antisettica delle ferite e proposta di un nuovo metodo. Memorie R. It. Veneto S.S.L.L.A.A. 19 (1876) 117-170.

${ }^{60}$ A. Menzel, Sul metodo di Lister. Gazzetta Medica Italiana-Lombardia 36 (1877) 241, 251-254.

61 Ibid., S. 252.

${ }^{62}$ Ibid., S.253. Man muß jedoch erklären, daß hier nicht die gesamte wissenschaftliche Produktion Menzels untersucht wurde. Es wurden nur einige der wichtigsten und eindrücklichsten Aspekte hervorgehoben.

${ }^{63}$ Op. cit. Anm. 10, S. 8.

${ }^{64}$ G. Ruggi, Dell'arte di medicare secondo il metodo Lister, Bologna 1879, S.18, 30, 31 und passim.

${ }^{65}$ Op. cit. Anm. 8, S. 199 (Brief an Prof. von Rindfleisch, 6. Dezember 1879).

${ }^{66}$ Ibid., S. 318 (Brief an Dr. Gersuny, 9. August 1886). 


\section{Summary}

The scientific achievements of the medical school of Vienna came to Italy especially through Padua and Triest. The surgery of Theodor Billroth was introduced by his highly gifted disciple Arthur Menzel (1844-1878). Menzel grew up in Triest. He studied eleven years in Vienna (1861-1872) and reached his habilitation; then he was chosen as a surgeon at the hospital of Triest. He died from tuberculosis with only 34 years.-Menzel's scientific work is described. Often he experimented with dogs. He worked about subcutaneous injection of food (which can be desirable before or after gastroenteric operations), the action of urine upon cellular tissue, the septic properties of fresh wound serum, bone operations, the treatment of fractures and sick joints, and the ovariotomy which was new. Early he used the perfected technics of anaesthesia, Lister's antisepsis, and Esmarch's artificial blood-emptiness. The surgery made great progresses, and Menzel stood in the front ranks of those who applicated them.

Prof. Dott. Loris Premuda

Istituto di Storia della Medicina

Via G. Falloppia, 50

I-35100 Padova 